\title{
NORMS ON UNITIZATIONS OF BANACH ALGEBRAS
}

\author{
A. K. GAUR AND Z. V. KOVÁR̆́íK
}

(Communicated by Palle E. T. Jorgensen)

\begin{abstract}
Equivalence of various norms on the unitization of a nonunital Banach algebra is established, with bounds (1 and $6 \exp (1))$ uniform over the class of such algebras. A tighter bound, 3 , is obtained in $C^{*}$-algebras for elements with Hermitian nonunital parts.
\end{abstract}

The algebra norm $\|\cdot\|$ on a nonunital Banach algebra $A$ can be extended to an algebra norm on the unitization $A^{+}$in many ways. Proposition 4.3 in [3] states that among these extensions, the $l_{1}$-norm

$$
\|\lambda e+a\|_{1}=|\lambda|+\|a\|
$$

is maximal and the operator norm

$$
\|\lambda e+a\|_{\mathrm{op}}=\sup \{\|\lambda x+a x\|:\|x\| \leq 1\}
$$

is minimal, provided that it does extend $\|\cdot\|$, i.e., that $\|\cdot\|$ is a regular (= operator) norm.

In the latter case, $A^{+}$is complete under both $\|\cdot\|_{1}$ and $\|\cdot\|_{\mathrm{op}}$, so by the "two-norm lemma" [2, II.2.5] these two norms are equivalent; the pure existence nature of the lemma does not yield an explicit bound $M$ in $\|\cdot\|_{1} \leq M\|\cdot\|_{\text {op }}$ and such a bound seems to depend on the algebra $A$.

The present theorem establishes uniform equivalence of the two unitization norms over the class of nonunital Banach algebras with regular norms.

Theorem. For every nonunital Banach algebra $A$ with unitization $A^{+}$and with regular norm, and for every $\lambda \in \mathbb{C}$ and $a \in A$, we have

$$
\|\lambda e+a\|_{\mathrm{op}} \leq\|\lambda e+a\|_{1} \leq(6 \exp 1)\|\lambda e+a\|_{\mathrm{op}} .
$$

If $A$ is a $C^{*}$-algebra, $a \in A$ is hermitian, and $\lambda$ is complex then

$$
\|\lambda e+a\|_{1} \leq 3\|\lambda e+a\|_{\mathrm{op}}
$$

and the constant 3 is best (minimal) possible.

Proof. In a general algebra $A$ with a regular norm, we have an extension of the classical inequality for the numerical radius $v(a)$ [1, Theorem 4.1]:

$$
v(a) \leq\|a\| \leq(\exp 1) v(a) .
$$

Received by the editors March 12, 1991 and, in revised form, May 13, 1991; presented at the AMS Baltimore Meeting (871) on January 10, 1992.

1991 Mathematics Subject Classification. Primary 46J99, 46J15.

Key words and phrases. Banach algebra, unitization, equivalent norms, Hermitian element. 
Without loss of generality, assume that $a \neq 0$. We know that the closure $K$ of the numerical range of $a$ in a nonunital algebra contains 0 ; our first task is to estimate $v(\lambda+a)$ from below: From the geometry of the complex plane we see that the diameter $d$ of the compact $K$ is realized as the distance $d=|\alpha-\beta|$ with $\alpha, \beta \in K$, and comparison with the special case $\lambda_{0}=-(\alpha+\beta) / 2$ leads to

$$
v(\lambda e+a)=\max \{|\lambda+\xi|: \xi \in K\} \geq \frac{1}{2} d .
$$

Also, since $0 \in K$, we have $d \geq v(a)$. Altogether,

$$
v(\lambda e+a) \geq \frac{1}{2} v(a) \text {. }
$$

Now we split estimates into cases $|\lambda| \leq 2\|a\|$ and $|\lambda|>2\|a\|$. The former case gives

$$
\frac{\|\lambda e+a\|_{\mathrm{op}}}{|\lambda|+\|a\|} \geq \frac{v(a) / 2}{2\|a\|+(\exp 1) v(a)} \geq \frac{v(a) / 2}{(3 \exp 1) v(a)}=\frac{1}{6 \exp 1}
$$

the latter case $|\lambda|>2\|a\|$ gives, using the triangle inequality and the fact that the fraction in the middle increases with $|\lambda|$,

$$
\frac{\|\lambda e+a\|_{\text {op }}}{|\lambda|+\|a\|} \geq \frac{|\lambda|-\|a\|}{|\lambda|+\|a\|} \geq \frac{1}{3} \text {. }
$$

We conclude that for all complex $\lambda$,

$$
\|\lambda+a\|_{1} \leq(6 \exp 1)\|\lambda+a\|_{\mathrm{op}} .
$$

Now the $C^{*}$-algebra case: The closure of the numerical range of a Hermitian $a$ is the smallest real interval $[\alpha, \beta]$ containing the spectrum of $a$, and for all complex $\lambda$ we have

$$
\begin{aligned}
\|\lambda e+a\|_{1} & =|\lambda|+\max (|\alpha|,|\beta|), \\
\|\lambda e+a\|_{\text {op }} & =\max (|\lambda+\alpha|,|\lambda+\beta|) .
\end{aligned}
$$

The expression to minimize is

$$
q(\lambda)=\frac{\max (|\lambda+\alpha|,|\lambda+\beta|)}{|\lambda|+\max (|\alpha|,|\beta|)} .
$$

Without loss of generality, we assume that $\alpha \leq 0<\beta$ and $\gamma=(\alpha+\beta) / 2 \geq 0$ (recall that 0 is in the spectrum of $a$ ); otherwise we replace $a$ with $-a$.

From now on, this is a problem about complex numbers. We split it into four cases:

(C1) $\lambda$ real,

(C2) $\lambda$ not real, $\Re \lambda>-\gamma$,

(C3) $\lambda$ is not real, $\Re \lambda<-\gamma$,

(C4) $\lambda$ not real, $\Re \lambda=-\gamma$.

In (C1) $q$ is continuous, piecewise monotone with breakpoints $-\beta, 0,-\gamma$, $-\alpha$, and respective values,

$$
\frac{\beta+|\alpha|}{2 \beta} \geq \frac{1}{2}, \quad \frac{\beta}{\beta}=1, \quad \frac{\beta+|\alpha|}{3 \beta-|\alpha|} \geq \frac{1}{3}, \quad \frac{\beta+|\alpha|}{\beta+|\alpha|}=1,
$$

and $q$ approaches 1 as $|\lambda| \rightarrow \infty$. The best we can say about $q$, therefore, is $q \geq \frac{1}{3}$, attained when $\alpha=0$. 
Case (C4). Write, for symmetry, $\alpha=\gamma-\rho \leq 0, \beta=\gamma+\rho>0$, so that $\rho=(\beta-\alpha) / 2$. Also, we substitute $p=-\gamma+\sqrt{\gamma^{2}+\nu^{2}}$ (note $p \geq 0$ ), so that $\nu^{2}=p^{2}+2 \dot{p} \gamma$. To prove that $Q(\nu)=q(-\gamma+i \nu) \geq \frac{1}{3}$, write

$$
\begin{aligned}
& Q(\nu)=\frac{|\lambda+\alpha|}{|\lambda|+\max (|\alpha|,|\beta|)}=\frac{\sqrt{\rho^{2}+\nu^{2}}}{\sqrt{\gamma^{2}+\nu^{2}}+\gamma+\rho}, \\
& \begin{array}{r}
Q^{2}(\nu)-\frac{1}{9}=\frac{9\left(\rho^{2}+p^{2}+2 p \gamma\right)-(p+\rho+2 \gamma)^{2}}{9(\rho+p+2 \gamma)^{2}} \\
=\frac{2(\rho-p-\gamma)^{2}+6(\rho+\gamma)(\rho-\gamma+p)}{9(\rho+p+2 \gamma)^{2}} \geq 0
\end{array}
\end{aligned}
$$

since both $\rho+\gamma>0$ and $\rho-\gamma+p=|\alpha|+p \geq 0$.

Cases (C2) and (C3). Except on the set $\{\lambda \mid \Re \lambda=-\gamma$ or $\lambda=0\}, q$ has a gradient

$$
\begin{aligned}
\nabla q(\lambda) & =\frac{(\lambda+\beta)(|\lambda|+\beta) /|\lambda+\beta|-|\lambda+\beta| \lambda /|\lambda|}{(|\lambda|+\beta)^{2}} \text { for } \Re \lambda>-\gamma, \\
& =\frac{(\lambda+\alpha)(|\lambda|+\beta) /|\lambda+\alpha|-|\lambda+\alpha| \lambda /|\lambda|}{(|\lambda|+\beta)^{2}} .
\end{aligned}
$$

Remark. The bound $6 \exp 1$ is not the best; by splitting at $(1+1 /(2 \exp 1))\|a\|$ instead of at $2\|a\|$ in the proof, we could reduce the bound $6 \exp 1$ to $1+$ $4 \exp 1$, but we suspect that even this can be improved.

\section{REFERENCES}

1. F. F. Bonsall and J. Duncan, Numerical ranges of operators on normed spaces and of elements of normed algebras, London Math. Soc. Lecture Notes Ser., vol. 2, Cambridge Univ. Press, Cambridge and New York, 1971.

2. N. Dunford and J. T. Schwartz, Linear operators, Part I, Interscience, New York, 1958.

3. A. K. Gaur and Z. V. Kováŕik, Norms, states and numerical ranges on direct sums, Analysis, Internat. Math. J. Anal. Appl. 11 (1991), 155-164.

Department of Mathematics, Duquesne University, Pittsburgh, Pennsylvania 158282

Department of Mathematics and Statistics, McMaster University, Hamilton, ONTARIO, CANADA L8S 4K1 